\title{
Missing the Distal-most [Z6] Impedance Channel? The Study Can Be Salvaged!
}

TO THE EDITOR: Combined multichannel intraluminal impedance and $\mathrm{pH}$ monitoring (MII-pH) is the preferred method for assessing gastroesophageal reflux (GER) in infants, children, and adults because it permits identification and examination of both acid $(\mathrm{pH}<4.0)$ and non-acid $(\mathrm{pH} \geq 4.0)$ (including weakly acidic and weakly alkaline) GER events. ${ }^{1}$ Occasionally, the tracing of a completed 24-hour impedance-pH study will be defective. For example, one or more impedance channels may be irreparably defective or missing completely (flat line). It is particularly problematic when the distal-most channel (Z6) is affected because (1) the extent of reflux bolus exposure (duration) is assessed in channel Z6, and (2) the autoscan of Medical Measurement System (MMS)/Laborie (Amsterdam, The Netherlands) will not work if Z6 is missing. Even if one were to go into the channel settings and de-select channel Z6, the autoscan would not use the Z5 channel to mark impedance-detectable reflux events. Consequently, assessment of temporal relationships cannot not be made until the tracing is manually scanned and edited to allow impedance-detected GER events to be recognized.

Figure 1A shows an impedance waveform pattern $(\mathrm{Z} 1-\mathrm{Z} 5)$ that illustrates a classic reflux event. In the absence of the distal channel (Z6), the MMS software algorithm will not capture any portions of the event by autoscan. However, the event can be captured manually (Fig. 1B) by executing the manufacturer recommended protocol of holding down the control key (Ctrl) and then left clicking at the bottom left (1), then the top left (2), and then the bottom right (3). Release of the Ctrl key then results in a partial capture (Fig. 1C) that can be completed using standard methods (Fig. 1D). Analyses in this manner allows the software algorithm to test the temporal associations between GER events and reported symptoms (Fig. 2). Assessments of bolus exposure can be estimated based on duration of channel (Z5)

Our rescue approach allows the clinician to derive meaningful information from the tracing without the need to subject the patient to further invasive testing. With the defective tracing featured in this article, we found (in a 4-month-old baby boy) that while the acid reflux index and total number $\mathrm{pH}$ probe-detected acid events $(\mathrm{pH}<4)$ were in the normal range for infants $(1.5 \%$ and 41 , respectively), ${ }^{2}$ impedance data showed that greater than $80 \%$ of both acid $(\mathrm{pH}<4,20 / 24)$ and non-acid $(\mathrm{pH} \geq 4$, 28/33) reflux events reached the proximal esophagus $(\mathrm{Z} 1$ and/or $\mathrm{Z} 2)$ and symptoms of cough were positively associated with both acid $(\mathrm{pH}<4)$ (symptom association probability $=96.5 \%)$ and non-acid $(\mathrm{pH} \geq 4)$ (symptom association probability $=100 \%$ ) GER.

Technically imperfect studies can most often (in hindsight) be linked to difficulties with inserting the catheter; twists and bends in the catheter during placement can cause breakages in the thin wires that run the length of the catheter. Technical errors can affect just parts of the study (that can be disregarded using artefact markers) or they can affect the entire study. Failure of the $\mathrm{pH}$ probe, for example, always requires retesting because assessment of the acid burden is a critical component of the impedance-pH report.

Frederick W Woodley ${ }^{1,2,3}$ ${ }^{1}$ Center for Motility Disorders, Nationwide Children's Hospital, Columbus, OH, USA; ${ }^{2}$ Division of Gastroenterology, Hepatology and Nutrition, Nationwide Children's Hospital, Columbus, OH, USA; and ${ }^{3}$ Department of Pediatrics, The Ohio State University College of Medicine, Columbus, OH, USA

1. Tutuian R, Castell DO. Review article: complete gastro-oesophageal reflux monitoring - combined $\mathrm{pH}$ and impedance. Aliment Pharmacol Ther 2006;24 (suppl 2):27-37.

2. Vandenplas Y, Goyvaerts H, Helven R, Sacre L. Gastroesophageal reflux, as measured by 24-hour $\mathrm{pH}$ monitoring, in 509 healthy infants screened for risk of sudden infant death syndrome. Pediatrics 1991;88:834-840.

Financial support: None.

Conflicts of interest: None. 

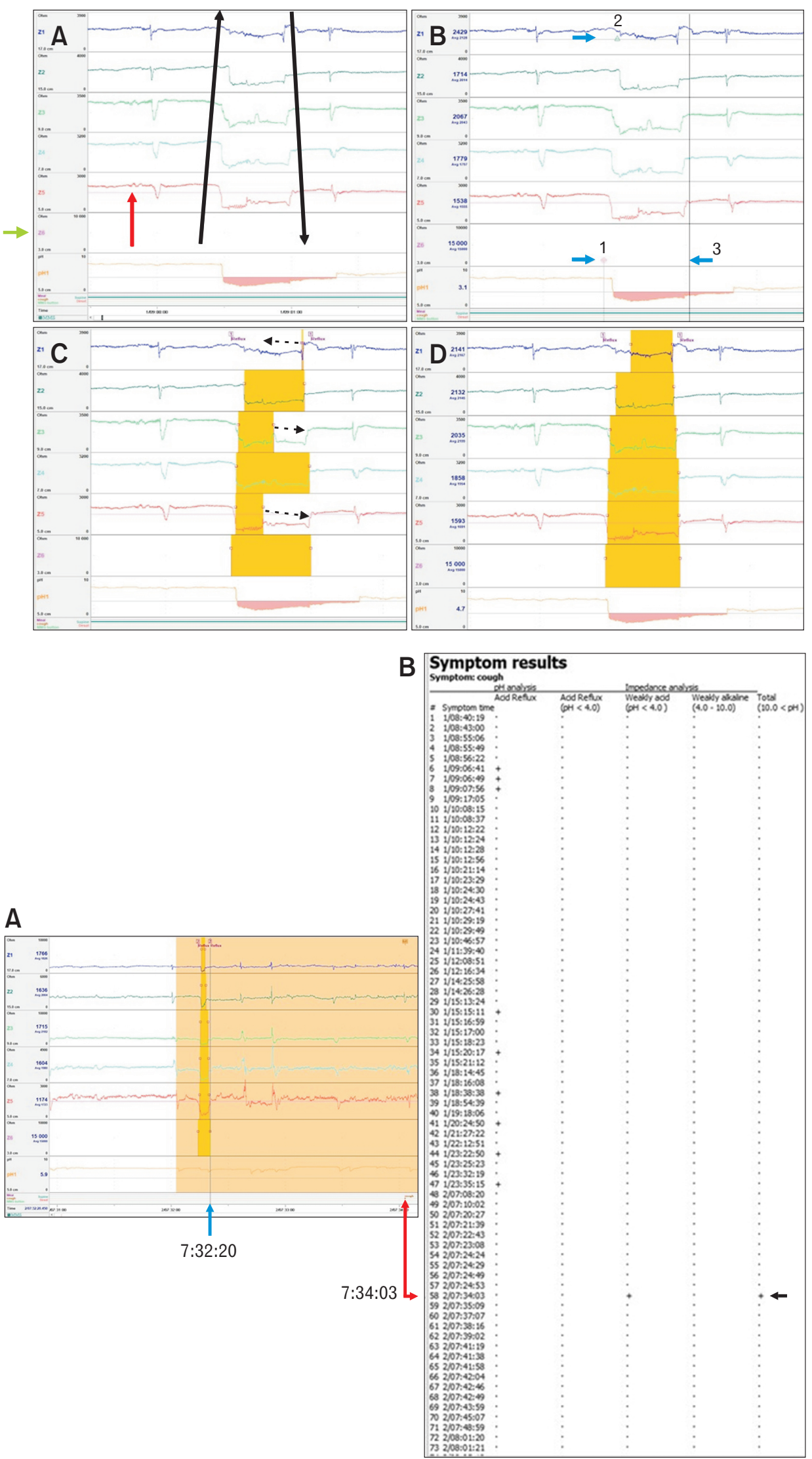

Figure 1. Tagging an impedance-detected GER event when the distal impedance channel (Z6) has malfunctioned. (A) Red arrow highlights the defective Z6 waveform which is evidenced by a flat-line. The black arrows indicate directional intraluminal flow of reflux within the esophagus. Green arrow highlights empty Z6 channel. (B) Numbers and short blue arrows mark the execution points (left clicks) in the protocol for manually tagging an impedance-detected event using the MMS system. (C, D) Panels illustrate the partial and completed tagging, respectively, of Z6-less impedance event. (C) The dotted-line arrows graphically illustrate the direction in which the tracing editor would right click and pull the appropriate lollipop; the left lollipop in channel $\mathrm{Z} 1$ would be pulled to the left and the right lollipops in $\mathrm{Z} 3$ and $\mathrm{Z} 5$ would be pulled to the right.

Figure 2. Non-acid reflux event is temporally associated with cough. (A) Graphically illustrates a non-acid reflux event that occurred (at 7:32:20) (blue arrow) just prior to a downstream cough (at 7:34:03) (red arrows). (B) Shows that the reflux event that occurred at 7 : $32: 20$ is temporally associated (black arrow) with a cough episode that occurred at 7:34:03, following an editing event described herein. 